\title{
THE USE OF DIATHERMY IN CASES OF GONOCOCCAL INFECTION
}

By E. P. CUMBERBATCH, B.M., B.Ch. (Oxon.), M.R.C.P., Medical Officer in Charge of the Electrical Department, St. Bartholomew's Hospital, and C. A. ROBINSON, M.B., D.M.R.E. (Cantab.), B.Ch. (Cantab.), Medical Officer in Charge of the Electrical Department of the West Middlesex Hospital; Clinical Assistant, Electrical Department, St. Bartholomew's Hospital.

Introduction.-The sensitivity of the gonococcus to heat has long been known. Cultures of the organism are destroyed by exposure to a temperature insufficient to harm the living tissues. Attempts have been made to treat gonococcal infection of the human subject by application of heat. Heated sounds have been introduced into the urethra in cases of gonorrhœa. Arthritis has been treated by hot fomentations or by currents of hot air. These methods of applying heat do not bring the infection to an end because they are unable to raise the temperature of the parts which lie at more than a slight depth below the surface. The living tissues conduct heat slowly. When the heat reaches the capillaries further conduction is hampered or prevented by the circulating blood. The moving fluid dissipates the heat by convection and prevents its spread to deeper layers.

When the diathermy current is used as the source of heat it is possible to raise the temperature of the tissues en masse. The heat is generated in the tissues themselves, and it is possible to produce it faster than it can be removed by convection. The temperature of the tissues traversed by the current can therefore be raised many degrees above normal, sufficient, if desired, to coagulate the tissue proteins.

Soon after the introduction of diathermy into this country by the late Dr. Lewis Jones the action of the new form of treatment was investigated by one of the present writers (E. P. C.) in cases of infective arthritis, and it was 


\section{BRITISH JOURNAL OF VENEREAL DISEASES}

found that gonococcal arthritis responded extremely well. The first series of cases was treated in I9I3 and I9I4. After the late war the investigation was continued and in I9I9 cases of gonococcal infection of other parts were treated by diathermy and the work was continued under a joint authorship. Infection of the joints, of the epididymis, testis and vas deferens, of the cervix uteri, Fallopian tubes and urethra in the female, of the prostate, seminal vesicles and urethra in the male, of the iris and of the supporting structures of the feet have been treated. At the present time the total number of cases treated has exceeded I5O. It is proposed, in the present article, to give a short account of the use of the diathermy current for heating the body, the influence of diathermy on the gonococcus and the infected tissues, and afterwards to describe in brief our methods and the results which we have obtained.

The Diathermy Current as a Means of Heating the Tissues. --In principle the use of the electric current for heating the tissues is simple. The tissues conduct electricity and their temperature can be raised just as non-living conductors are heated by passing a current through them. To heat the tissues the current must be of considerable strength and the ordinary currents, such as the galvanic, faradic and sinusoidal, cannot be used for the purpose. If the current is one which oscillates with a frequency of a million or more times per second it is unable to stimulate the excitable tissues or produce chemical (electrolytic) changes in the tissues. It can therefore be passed through the body and its strength increased to a value sufficient to heat the tissues to the degree desired. The only sensation produced is that of heat.

Currents which oscillate with this frequency do not behave like other currents in regard to their path and distribution between the electrodes. Their precise path in that most complex of all conductors, viz. the human body, is uncertain. The use of thermometers gives some clue as to the distribution of the heat which they generate. Thermometers, however, can only be inserted in a few regions of the body, and they do not always give a trustworthy measure of the heat generated by the current. In devising methods of heating infected parts by the diathermy current we have been compelled to rely on clinical results when deciding the value of any method of applica- 


\section{BRITISH JOURNAL OF VENEREAL DISEASES}

tion and to rely on the patient's sensations in order to avoid a dangerous degree of heat. This reliance has not been misplaced, and the use of the methods which will be described later will not cause burns, provided that the operator is fully familiar with the application of diathermy to all parts of the body. Such familiarity can be gained only by long experience.

The Sensitivity of the Gonococcus to Heat.-With regard to the lethal temperature for the gonococcus different figures have been given by different observers. Most of the figures agree, however, in showing that the organism can be destroyed at a temperature which is not high enough to damage the living tissues. Santos showed that the gonococcus could be destroyed at $109^{\circ} \mathrm{F}$. after an exposure of seventy-six minutes. At a temperature of III $\cdot 2^{\circ} \mathrm{F}$. it could be killed by an exposure of fifty-four minutes. At $\operatorname{II} 3^{\circ} \mathrm{F}$. the exposure could be reduced to thirty-seven minutes to produce the same result. These figures show the importance of another factor, that of the duration of exposure. If the exposure is short a higher temperature is required, and vice versâ. The figures obtained by various observers, while showing that the treatment of gonococcal infection by heat is rational, give little clue as to the temperature to which the infected parts should be raised or the length of time for which they should be heated.

In the living tissues the conditions and surroundings are quite different from those present in non-living media. In the latter the only factors concerned in the destruction of the gonococci are those of temperature and time. In the living tissues there are agents hostile to the life and growth of invading organisms, and it is necessary to consider the influence of the heat on the self-protective mechanism of the invaded tissues as well as on the infecting gonococci. We believe that diathermy, in addition to its direct action on the gonococci, increases the power of the tissues to exterminate the infecting organisms.

We have often found that temperatures lower and exposures shorter than those given by Santos and others were able to bring metastatic infection to an end, while evidence of infection of the primary foci disappeared. Heating the infected cervix uteri to $I I 4^{\circ} \mathrm{F}$. for ten minutes and repeating the treatment twice at three-day intervals has often been sufficient to cure or arrest metastatic 


\section{BRITISH JOURNAL OF VENEREAL DISEASES}

infection and procure apparent (and probably actual) freedom of the primary foci from gonococci.

In a certain number of cases of gonococcal arthritis in women we have been unable to heat the cervix uteri to $I I 4^{\circ} \mathrm{F}$. Infection of the Fallopian tubes was present in these cases, and we found that attempts to heat the cervix to the temperature mentioned caused severe pain in the pelvis. The current could not be increased to more than half the strength necessary to produce a temperature of II $4^{\circ} \mathrm{F}$. The effects on the arthritis were, however, as good as those obtained in other cases in which the cervix was heated to $\mathrm{II}_{4}^{\circ} \mathrm{F}$. This has been observed both in children and adults. The prostate and seminal vesicles cannot be heated to a temperature as high as $I I 4^{\circ} \mathrm{F}$. Yet we have been able, in all our cases of gonococcal infection of the joints in men, to cure or arrest the arthritis by applying diathermy to the vesicles and prostate.

It appears, then, that diathermy acts in two ways. It diminishes the vitality of the gonococci or, in certain situations where the highest temperature consistent with safety can be attained, destroys them. It also increases the power of the tissues to remove the infecting organisms. In other words, diathermy has a direct and an indirect action on the goncoocci. To procure the direct action high temperatures are required, and relatively short exposures. High temperatures can be produced only in parts which can be brought into direct contact with the electrodes, as, for example, the urethra and cervix. We have found, however, that a temperature of $\mathrm{II} 4^{\circ} \mathrm{F}$. is the maximum that should be used. We have always noted that the sensation of heat changes to that of pain somewhere between $\operatorname{II} 4^{\circ}$ and $I I 5^{\circ} \mathrm{F}$. Although temperatures a few degrees higher can be reached without damage to the tissues we think it unsafe to increase the current to the strength at which pain is produced. It is also inadvisable, because the infected tissues may lose their self-protective power when heated to a temperature sufficient to cause pain.

In regions where high temperatures cannot be produced, as for example in the prostate and seminal vesicles, it is probable that diathermy does not destroy the gonococci directly, but diminishes their vitality and increases the power of the infected tissues to exterminate the weakened organisms. 


\section{BRITISH JOURNAL OF VENEREAL DISEASES}

We are unable to state the temperature and time of exposure which will increase the self-protective powers of the tissues to their maximum. When treating gonococcal infection by diathermy we have obtained high temperatures and given short exposures when the infected parts can be brought into direct contact with the electrodes. When the infected parts cannot be brought into direct contact, and a temperature of $\mathrm{II}_{4}^{\circ} \mathrm{F}$. cannot be attained, we have continued the applications for longer times.

Treatment of Gonococcal Infection in the Female.Whatever region is infected, and whether metastatic infection is present in addition to the primary infection or not, we now commence the treatment by applying diathermy to the urethra and cervix uteri. These parts are treated whether gonococci are found in them or not, and whether the parts have already been treated by other methods or not. In married women we have adopted the following method after trial of many others. The urethra is first subjected to diathermy. A bougie electrode is inserted, and the circuit is completed by means of a belt electrode of sheet lead which is secured around the pelvis. The current is gradually increased until the patient begins to feel that the sensation of pain is replacing that of heat. The current is now reduced slightly until the pain disappears, and it is kept at this strength for ten minutes. We have devised electrodes for the urethra with thermometers attached, but we prefer the simple bougie electrode.

After treating the urethra the cervix is subjected to diathermy. A bougie electrode is inserted into the canal after passing a speculum per vaginam, and the circuit is completed by the belt electrode as before. Since the cervix is insensitive to heat and pain the patient's sensations afford no guide to the strength of current which should be used. In our earlier work we were unable to judge the correct strength of current. In some cases the cervix was insufficiently heated, and burns were produced in others.

Methods of measuring the temperature were then considered. The introduction of a thermometer into the cervix after the latter has been subjected to a trial of diathermy does not give an accurate measure of the heat attained during the diathermy because the temperature begins to fall directly after the cessation of the current. We have devised an electrode containing a thermometer, 


\section{BRITISH JOURNAL OF VENEREAL DISEASES}

but the use of such an instrument is open to objection. A thermometer enclosed in a metal case indicates a temperature which is two or three degrees below that reached in those parts of the cervix below the mucous membrane of the canal, and the difference between the two values is not always the same. We have been able in a few cases to insert a thermometer in the cervix by the side of a bougie electrode. When this is done, however, a complication arises from the fact that a mercury thermometer when not enclosed in a metal case acts as a condenser electrode and gives a false reading.

The thermo-electric couple was considered but was not adopted because an instrument of this kind is impracticable for clinical work. It requires too much attention. This objection has particular weight when two or three patients are being treated at the same time, as is usually the case in the Electrical Department of St. Bartholomew's Hospital. •

We have therefore adopted the following method of procuring the correct temperature, viz. II $4^{\circ} \mathrm{F}$. in the cervix. The electrode used for the urethra and the strength of current applied to this part furnish the indications for the strength of current that should be applied in order to produce this temperature in the cervix.

The current used for the urethra is noted and the area of contact which the urethral electrode makes with the mucous membrane is calculated. The length of insertion of the cervical electrode and the diameter of the latter are noted. The area of contact with the mucous membrane of the canal of the cervix is calculated. The current for the cervix, compared with that applied to the urethra is reduced in direct proportion to the smaller area of contact made by the cervical electrode. When both electrodes have the same diameter the length of insertion only need be noted. If the length of insertion into the cervix is half the length of insertion into the urethra the current applied to the cervix should be one half that applied to the urethra.

By means of electrodes containing thermometers, the necessary corrections having been made, we have repeatedly found that the current calculated in the way described produces the same temperature in the cervix as in the urethra. A detailed description of the method which we now use will be found in a book by the present writers 


\section{BRITISH JOURNAL OF VENEREAL DISEASES}

entitled "Gonococcal Infection and its Treatment by Diathermy." This book will shortly be published by William Heinemann, Ltd.

Having obtained the current which produces the desired temperature of the cervix and urethra it must not be thought that the application of the same current on subsequent occasions will produce the same temperatures, even in the same patient. The temperature produced by the current depends on the resistance of the pelvic tissues. The resistance is not constant, but varies from time to time according to the amount of blood in the pelvic organs, and the presence or absence of urine in the bladder and of fæces and gas in the intestine. Increase of the resistance will cause the same current to produce a higher temperature, and vice versâ. Increase of the blood supply lowers the resistance; less heat is generated and more heat is removed by convection.

At each treatment, therefore, the current which produces the standard temperature of $\mathrm{II}_{4}^{\circ} \mathrm{F}$. in the urethra must be ascertained afresh. It will be found to vary from time to time.

At each session of treatment diathermy is applied first to the urethra for ten minutes and then to the cervix for the same time. The treatment is repeated twice weekly and from three to five sessions are held. It is seldom necessary to give further treatment to these parts.

When a vaginal speculum cannot be introduced diathermy can be applied to the cervix in the following way. An electrode is passed into the rectum. The electrode which we have devised for the purpose of applying diathermy to the cervix by the rectal route consists of a hollow metal case, eight inches long, oval in cross section and half an inch wide at its broadest part. In its interior a tube is attached for holding a thermometer. One end of the electrode is rounded and closed. This end is passed through the anus and the electrode is passed for four inches into the rectum. The end which lies outside the body is depressed on to the couch on which the patient lies by means of a sand bag. The circuit is completed by means of the belt electrode already described. When the current is passing the patient feels a sensation of heat within the rectum. The current is increased gradually until she feels a sensation of pain; it is then slightly reduced. We have found that the sensation changes 


\section{BRITISH JOURNAL OF VENEREAL DISEASES}

from heat to pain at $I I 4^{\circ} \mathrm{F}$. This temperature is reached in the rectal wall. The cervix, not being in contact with the electrode, does not reach so high a temperature. We applied diathermy to the cervix by the rectal route in a multipara and found that a thermometer inserted into the canal of the cervix recorded a temperature of $109^{\circ} \mathrm{F}$. when the rectal temperature was $I I 4^{\circ} \mathrm{F}$.

The current is allowed to pass for twenty minutes and the treatment is repeated twice weekly until three to five sessions have been held. The treatment of the cervix is always preceded by treatment of the urethra.

We have been able to apply diathermy by the rectal route to children suffering from gonococcal vaginitis and urethritis. When they have been accustomed to vaginal douching an electrode may be introduced into the vagina. When applying diathermy to children we do not attempt to obtain high temperatures, but we prolong the exposure.

In the next number of the Journal we shall describe our methods of applying diathermy for the treatment of gonococcal infection in the male. The results which we have obtained in both sexes will be given, and it will be shown that gonococcal infection of the joints can be cured or arrested by the application of diathermy to the primary foci of infection, and that the same treatment appears to free the latter from the infecting gonococci.

(To be continued.) 